DOI: 10.12957/demetra.2017.28085

\title{
Relação entre a presença de sintomas de ansiedade e estado nutricional em idosos residentes de Florianópolis-SC
}

\section{Relationship between anxiety symptoms and nutritional status in elderly residents of Florianópolis-SC}

Patrícia Pan de Matos'

Sabrina Vilela Ribeiro'

Júlia Dubois Moreira?

1 Universidade Federal de Santa Catarina, Departamento de Nutrição, Centro de Ciências da Saúde. Florianópolis-SC, Brasil.

Correspondência / Correspondence Julia Dubois Moreira

E-mail: juliamoreira@gmail.com

\section{Resumo}

Objetivo: Investigar a associação entre a presença de sintomas de ansiedade e estado nutricional, estimado pelo índice de massa corporal (IMC), em idosos residentes de Florianópolis/SC. Métodos: Estudo transversal realizado com 146 idosos frequentadores do Núcleo de Estudos da Terceira Idade (NETI) - UFSC/Florianópolis. Para avaliar presença de sintomas de ansiedade, aplicou-se o Geriatric Anxiety Inventory (GAI). O estado nutricional foi avaliado por meio do IMC, classificado de acordo com os parâmetros da World Health Organization (WHO, 2008) e de Lipschitz (1994). Para a análise estatística, utilizou-se o software Stata 11.0. Testes qui-quadrado e correlação de Pearson foram usados para aferir a relação entre IMC e presença de ansiedade. Utilizando regressão logística, verificou-se o risco para ansiedade segundo as categorias de IMC. Foi considerado $\mathrm{P}<0,05$ para significância. Resultados: A maioria dos participantes foram mulheres $(88,36 \%)$ e com idade menor que 80 anos $(93,15 \%)$; 39 (26,71\%) obtiveram pontuação $>10$, classificando-os como ansiosos. Houve associação da presença de sintomas de ansiedade com o IMC quando usados os critérios de classificação l da WHO $(\beta=13,09 ; \mathrm{P}=0,004)$, mas não quando usados os critérios de Lipschitz $(\beta=3,78 ; P=0,151)$. Quando aplicada a regressão logística bruta, houve maior risco para ansiedade quando IMC $\geq 30(\mathrm{OR}=2,67 ;$ IC95\% 1,2375 - 5,7621; $\mathrm{P}=0,012)$. No modelo ajustado pela idade, o risco aumentou $(\mathrm{OR}=2,91$; IC95\% 1,3257 6,4047; P=0,008). Conclusão: Existe relação entre a presença de ansiedade e obesidade em idosos. Idosos obesos têm quase 3 vezes mais risco de serem ansiosos que as demais categorias de IMC.

Palavras-chave: Ansiedade. Idosos. Estado Nutricional. Índice de Massa Corporal. 


\section{Abstract}

Objective: To investigate the association between the presence of anxiety symptoms and nutritional status estimated by the body mass index (BMI) in the elderly. Methods: Cross-sectional study that evaluated 146 elderly people attending the Third Age Research Center (NETI/UFSC, Florianópolis, Brazil). To evaluate the presence of anxiety symptoms, the Geriatric Anxiety Inventory (GAI) was applied. Nutritional status was evaluated by the BMI [WHO (2008) and Lipschitz (1994)]. The Stata 11.0 software was used for analysis, and the chi-square test and Pearson's correlation were calculated for BMI and presence of anxiety. Logistic regression was applied to evaluate odds ratio (OR) for anxiety risk in each BMI category. Results: The majority of individuals were women $(88.36 \%)$ and their age was below 80 years $(93.15 \%)$. Thirty-nine individuals $(26.71 \%)$ had a score $>10$ points, hence they were classified as anxious. There was association between presence of anxiety symptoms with BMI when using the categories recommended by WHO $(\beta=13.09$, $\mathrm{P}=0.004)$, but not when using the categories recommended by Lipschitz $(\beta=3.78, P=0.151)$. When regression logistics was applied, there was increased risk for anxiety with BMI $\geq 30$ (OR $=2.67 ; 95 \%$ CI 1.2375 to $5.7621 ; \mathrm{P}=0.012)$. In the adjusted model by age, this risk remained and increased $(\mathrm{OR}=2.91 ; 95 \% \mathrm{CI}$ 1.3257 to $6.4047 ; \mathrm{P}=0.008)$. Conclusion: There is a relationship between presence of anxiety and obesity in the elderly population. Elderly obese individuals had nearly 3 times the risk of being anxious than the other BMI categories.

Keywords: Anxiety. Elderly. Nutritional Status. Body Mass Index.

\section{Introdução}

Os transtornos de humor e ansiedade são frequentes em idosos e sua prevalência vem aumentando na população mundial., ${ }^{1,2}$ Dados da pesquisa americana National Survey on Drug Use and Health, que foi conduzida pelo Substance Abuse and Mental Health Service Administration (SAMHSA) entre os anos 2008 e 2012, mostraram que os transtornos mais prevalentes em indivíduos com 50 anos ou mais foram os transtornos depressivos, um ou mais transtorno de ansiedade (ansiedade generalizada, transtorno de pânico) e abuso de álcool. ${ }^{1}$ No Brasil, o Caderno de Atenção Básica "Envelhecimento e saúde da pessoa idosa", do Ministério da Saúde, traz a depressão como 
o transtorno de humor mais prevalente na população idosa (de 4,7\% a 36,8\%, dependendo da escala e critérios de diagnóstico utilizados). ${ }^{3}$ No entanto, são escassos dados populacionais acerca de outros diagnósticos de transtornos de humor vivenciados na população, especialmente entre idosos, o que torna difícil a elaboração de políticas públicas voltadas para esta necessidade.

Estudos mostram que os transtornos de ansiedade figuram entre os problemas de saúde mental mais presentes na vida do idoso. ${ }^{3}$ Porém, há uma diferença na forma de apresentação destes transtornos em pessoas idosas, muitas vezes sendo de difícil identificação, o que pode resultar em atraso por busca de ajuda especializada. ${ }^{4}$ Nesse sentido, a rede de Atenção Psicossocial vinculada ao Governo do Estado de Santa Catarina montou o Protocolo para Acolhimento e Tratamento dos Transtornos de Pânico e Ansiedade Generalizada, tendo em vista a magnitude da busca por serviços de saúde com esta demanda. ${ }^{5}$

O envelhecimento, apesar de ser um processo natural, submete o organismo a diferentes alterações morfológicas, fisiológicas, bioquímicas e psicológicas, que levam a uma redução da capacidade de adaptação do indivíduo ao meio ambiente, resultando em repercussões nas suas condições de saúde e nutrição. ${ }^{4} \mathrm{O}$ estado nutricional adequado é um dos fatores que contribuem para o processo de envelhecimento com saúde, ${ }^{5}$ contudo, o panorama da obesidade está aumentando na população idosa. ${ }^{6,7}$

A obesidade é uma doença multifatorial, tendo como seus principais determinantes fatores ambientais (sedentarismo, alimentação), de estilo de vida (escolaridade, renda familiar, paridade, estado civil), genéticos (polimorfismos), iatrogênicos (uso de medicamentos que contribuem para o ganho de peso) e também comportamentais (estresse, saúde mental). ${ }^{8}$ Estudos têm evidenciado que o estado nutricional pode influenciar na saúde mental e no desenvolvimento de transtornos de humor. Simon et al., com uma amostra representativa de adultos nos Estados Unidos ( $\mathrm{n}=9125)$, encontraram associação entre obesidade e transtornos de humor, como depressão, transtorno bipolar e transtorno do pânico, concluindo que indivíduos obesos são, aproximadamente, $25 \%$ mais propensos a transtornos de humor e ansiedade. ${ }^{9}$ Existem algumas evidências que sugerem que níveis elevados de estresse, frequentemente presentes em situação de ansiedade, estão associados com o aumento do apetite e ingestão de alimentos mais calóricos, o que pode contribuir para o ganho de peso. ${ }^{10}$

São escassos na literatura estudos que avaliaram a presença de transtornos de humor, em especial, a ansiedade, na população idosa e sua relação com o estado nutricional. Nesse sentido, o objetivo deste estudo é investigar a associação entre a presença de sintomas de ansiedade e estado nutricional em idosos. 


\section{Método}

O projeto de pesquisa seguiu os critérios da Resolução 466, de 12 de dezembro de 2012, e foi aprovado pelo Comitê de Ética em Pesquisa com Seres Humanos (CEPSH) da UFSC (protocolo no 485.331, de 2014).

Este estudo transversal foi realizado com 146 idosos, de ambos os sexos, frequentadores das atividades do Núcleo de Estudos da Terceira Idade (NETI) - UFSC/Florianópolis, Santa Catarina. A amostra deste estudo foi não probabilística por conveniência. Como critérios de Inclusão, os indivíduos deveriam apresentar idade igual ou superior a 60 anos, de ambos os sexos, que assinaram o Termo de Consentimento Livre e Esclarecido. Como critérios de Exclusão, os Indivíduos que apresentassem algum membro amputado, edema ou ascite, ou outro fator que interferisse no resultado da avaliação do estado nutricional pela aferição de peso e altura.

A coleta de dados iniciou-se em junho de 2016, sendo as pesquisadoras de campo acadêmicas do curso de Nutrição da Universidade Federal de Santa Catarina. O projeto não apresentou riscos para os indivíduos que aceitaram participar da pesquisa. Os dados encontrados nesta pesquisa foram mantidos em anonimato, e o participante recebeu um número de identificação, caso tivesse interesse em saber o resultado de sua participação. Além disso, poderia contatar os responsáveis pela pesquisa a qualquer momento por meio de telefone ou endereço eletrônico, para saber o andamento da pesquisa e/ou obter esclarecimentos.

Para verificar a presença de sintomas de ansiedade, foi utilizado o Geriatric Anxiety Inventory (GAI), elaborado por Pachana et al., ${ }^{11}$ adaptado para o português e validado por Martiny et al. ${ }^{12}$ Este é um questionário autoaplicado, que evita qualquer tipo de constrangimento em responder, e foi utilizado pelas pesquisadoras em circunstâncias em que o indivíduo apresentava dificuldades para leitura. O instrumento é composto por 20 afirmações sobre situações que podem causar ansiedade, e o respondente tem que assinalar entre as duas opções para cada afirmativa, concordo ou discordo. ${ }^{13}$ Segundo os autores do instrumento, pessoas que obtiveram pontuação maior ou igual a 10, foram classificadas como ansiosas, conforme definição do Manual Diagnóstico e Estatístico de Transtornos Mentais (DSM-IV-TR). ${ }^{14}$

Após o preenchimento do questionário, foi aferido o peso (kg) e estatura (m) de cada indivíduo. Para a medida do peso, foi utilizada balança portátil digital com função tara, capacidade de até $200 \mathrm{~kg}$ e precisão de $50 \mathrm{~g}$ da marca Wiso modelo W920. A estatura foi aferida com antropômetro portátil, com plataforma anexa com extensão de 2 metros e precisão de $1 \mathrm{~mm}$ da marca Alturexata .Posteriormente, foi calculado o IMC para a classificação do estado nutricional pelos pontos de corte preconizados pela WHO, que recomenda pontos de corte para definição de baixo peso (índice de massa corporal <18,5 kg/m²), eutrofia (índice de massa corporal entre 18,5 e 24,9 kg/ 
$\mathrm{m}^{2}$ ), sobrepeso (índice de massa corporal entre 25 e $29,9 \mathrm{~kg} / \mathrm{m}^{2}$ ) e obesidade (índice de massa corporal $\left.>30 \mathrm{~kg} / \mathrm{m}^{2}\right),{ }^{15}$ e por Lipschitz, que recomenda pontos de corte para definição de baixo peso (índice de massa corporal $<22 \mathrm{~kg} / \mathrm{m}^{2}$ ), eutrofia (índice de massa corporal entre 22 e $27 \mathrm{~kg} / \mathrm{m}^{2}$ ) e excesso de peso (índice de massa corporal $\left.>27 \mathrm{~kg} / \mathrm{m}^{2}\right){ }^{16}$ preconizado pelo Ministério da Saúde. ${ }^{17}$

Finalizada a coleta de dados, estes foram tabulados no software Excel 2010, transferidos pelo softwear StatTranfer para posterior análise no software Stata 11.0. Os dados foram apresentados em frequência absoluta e relativa, e foram avaliados quanto à simetria pelo teste de Shapiro-Wilk. Os dados simétricos foram expressos em média \pm desvio-padrão, e os dados assimétricos foram expressos em mediana e intervalo interquartil. Para verificar a correlação entre o peso, IMC, idade e a pontuação obtida na escala de ansiedade, foram feitos regressão linear simples e teste de correlação de Pearson. O teste T de Student foi utilizado para aferir a diferença entre os dados antropométricos dos indivíduos com e sem ansiedade. Para verificar a associação entre as variáveis categóricas (IMC, idade e presença de ansiedade), foi aplicado o teste qui-quadrado. Quando houve associação (IMC e presença de ansiedade), foi realizada a regressão logística para cálculo da razão de odds (RO), tanto a análise bruta, quanto ajustada para idade, tendo a ansiedade como desfecho e as categorias de IMC como variável independente.

\section{Resultados}

Entre os idosos frequentadores do NETI, 146 indivíduos que preencheram os critérios de participação aceitaram fazer parte deste estudo. Na Tabela 1 estão expostas as características da população avaliada. A maior parte dos indivíduos, 129 (88,35\%), foi do sexo feminino, e 136 $(93,15 \%)$ da amostra tinham idade inferior a 80 anos. Entre os avaliados, 39 (26,71\%) obtiveram pontuação maior que 10 pontos, classificando-se como ansiosos. Das pessoas classificadas como ansiosas, $30(76,92 \%)$ apresentaram IMC acima de 24,9 kg/m², ou seja, excesso de peso, sendo 12 $(30,77 \%)$ com sobrepeso e $18(46,15 \%)$ com obesidade.

A Tabela 2 mostra a diferença das médias de peso e IMC dos indivíduos com e sem ansiedade. Observou-se que indivíduos ansiosos têm média de IMC superior aos indivíduos sem ansiedade. 
Tabela 1. Característica da população de idosos avaliada. Florianópolis - SC, 2016.

\begin{tabular}{|c|c|c|c|}
\hline & & Frequência (n) & $\%$ \\
\hline & Total & 146 & 100 \\
\hline \multirow[t]{2}{*}{ Sexo } & Masculino & 17 & 11,64 \\
\hline & Feminino & 129 & 88,36 \\
\hline \multirow[t]{2}{*}{ Idade } & $>80$ anos & 136 & 93,15 \\
\hline & $\geq 80$ anos & 10 & 6,85 \\
\hline \multirow[t]{2}{*}{ Antropometria } & Peso (kg) & $70,61 \pm 13,97 *$ & - \\
\hline & Altura (m) & $1,58 \pm 7,66^{*}$ & - \\
\hline \multirow[t]{5}{*}{ IMC (WHO, 2008) } & & $28,0 \pm 4,80^{*}$ & \\
\hline & Desnutrição & 2 & 1,37 \\
\hline & Eutrofia & 39 & 26,71 \\
\hline & Sobrepeso & 61 & 41,78 \\
\hline & Obesidade & 44 & 30,14 \\
\hline \multirow[t]{3}{*}{ IMC (Lipschitz, 2004) } & Baixo peso & 18 & 7,53 \\
\hline & Eutrofia & 52 & 35,62 \\
\hline & Excesso de peso & 83 & 56,85 \\
\hline \multirow[t]{2}{*}{ Presença de ansiedade } & $\operatorname{Sim}$ & 39 & 26,71 \\
\hline & Não & 107 & 73,29 \\
\hline
\end{tabular}

*Peso, altura e IMC foram apresentados como média \pm DP.

Tabela 2. Peso e IMC estratificados por presença de ansiedade em idosos de Florianópolis - SC, 2016.

\begin{tabular}{cccc}
\hline & Ansioso & Não ansioso & P-valor \\
\hline Peso $(\mathrm{kg})$ & $72,98 \pm 16,20$ & $69,75 \pm 13,04$ & 0,271 \\
IMC $\left(\mathrm{kg} / \mathrm{m}^{2}\right)$ & $29,30 \pm 5,86$ & $27,53 \pm 4,29$ & 0,049 \\
\hline
\end{tabular}

*Peso e IMC foram apresentados como média \pm DP. 
Segundo o teste de correlação de Pearson, houve uma correlação fraca e não estatisticamente significativa entre IMC, peso corporal e o escore de ansiedade (para IMC $\mathrm{r}=0.1157, \mathrm{p}=0,164$; para peso $\mathrm{r}=0,063, \mathrm{p}=0,447)$.

Houve associação entre IMC e presença de ansiedade, utilizando a classificação da WHO $(\beta=13,09 ; \mathrm{P}=0,004)$. Para os parâmetros de Lipschitz, preconizados pelo MS, não foi identificada associação $(\beta=3,78 ; P=0,151)$. Não houve associação entre idade e presença de ansiedade $(\beta=1,53$; $\mathrm{P}=0,2)$.

Na Tabela 3, estão expostas as RO para presença de ansiedade e IMC (na forma contínua e categorizada pelos critérios preconizados pela WHO). Na análise bruta, observa-se que os indivíduos classificados como obesos apresentam risco 2,67 vezes maior de apresentar ansiedade $(\mathrm{P}=0,012)$. Este risco se torna ainda maior quando a análise foi ajustada para idade $(\mathrm{RO}=2,91$; IC95\% 1,3257 - 6,4047; $\mathrm{p}=0,008)$.

Tabela 3. Associação entre a presença de ansiedade e as categorias de IMC (WHO, 2008) em idosos de Florianópolis - SC, 2016.

\begin{tabular}{lccc}
\hline \multicolumn{1}{c}{ IMC $(\mathrm{WHO}, 2008)$} & Razão de odds & IC 95\% & P-valor \\
\hline Análise bruta & & & \\
IMC (contínua) & 1,08 & $1,0055-1,1752$ & 0,036 \\
Eutrofia $\left(18,5-24,9 \mathrm{~kg} / \mathrm{m}^{2}\right)$ & 0,5126 & $0,2050-1,2820$ & 0,153 \\
Sobrepeso $\left(25,0-29,9 \mathrm{~kg} / \mathrm{m}^{2}\right)$ & 0,5260 & $0,2413-1,1466$ & 0,106 \\
Obesidade $\left(\geq 30 \mathrm{~kg} / \mathrm{m}^{2}\right)$ & 2,6703 & $1,2375-5,7621$ & 0,012 \\
\hline Análise ajustada $(i d a d e)$ & & & \\
IMC (contínua) & 1,09 & $1,0081-1,1798$ & 0,031 \\
Eutrofia $\left(18,5-24,9 \mathrm{~kg} / \mathrm{m}^{2}\right)$ & 0,4827 & $0,1910-1,2199$ & 0,124 \\
Sobrepeso $\left(25,0-29,9 \mathrm{~kg} / \mathrm{m}^{2}\right)$ & 0,5246 & $0,2396-1,1486$ & 0,107 \\
Obesidade $\left(\geq 30 \mathrm{~kg} / \mathrm{m}^{2}\right)$ & 2,9139 & $1,3257-6,4047$ & 0,008 \\
\hline
\end{tabular}


Das questões presentes no questionário GAI, quatro apresentaram destaque na quantidade de concordâncias por parte dos participantes. As afirmações com maior aquiescência foram: "Eu me preocupo em grande parte do tempo" - 85 pessoas (58,21\%); "Eu penso que sou preocupado" - também com 85 pessoas $(58,21 \%)$; "Meus próprios pensamentos com frequência me deixam ansioso" - com 68 (46,57\%) indivíduos; e "Eu acho que minhas preocupações interferem em minha vida” - também com 68 $(46,57 \%)$ concordantes. Apesar de nem todos os idosos que concordaram com essas alternativas terem sido classificados como ansiosos segundo o ponto de corte preconizado pelos autores da escala, eles assinalaram questões pertinentes a sintomas de ansiedade, uma vez que se consideraram pessoas que se preocupam em grande parte do tempo e por vezes demonstrarem ansiedade por preocupar-se. Isso mostra que muitos apresentam sintomas, mas não necessariamente o transtorno de ansiedade.

\section{Discussão}

O presente estudo avaliou a relação entre a presença de ansiedade e o estado nutricional de um grupo de idosos em Florianópolis, SC. Observou-se que há associação entre o IMC e a presença de ansiedade, sendo os obesos os com maior risco de apresentarem este transtorno de humor.

Uma revisão da literatura com o objetivo de avaliar os efeitos do avançar da idade na expressão clínica de ansiedade estima que o número de pessoas com idade superior a 65 anos, classificadas com presença de transtorno de ansiedade, representa aproximadamente $3,2 \%$ a $14,2 \% .{ }^{18}$

No presente estudo, 26,71\% dos idosos foram classificados como ansiosos. Essa porcentagem representa valor semelhante e superior a achados na revisão.

Em relação ao panorama do estado nutricional da população idosa brasileira, um estudo que utilizou os dados da Pesquisa de Orçamentos Familiares (POF 2008-2009) ${ }^{7}$ concluiu que o sobrepeso demonstrou prevalência bastante expressiva em idosos de todas as cores/raça, sendo mais comum entre idosas (41,9\%). Foi constatado que as maiores prevalências de obesidade residem nas regiões Sul $(45,1 \%)$ e Sudeste $(38,3 \%)$ e no meio urbano (39\%). Houve uma relação inversamente proporcional do sobrepeso com o aumento da faixa etária e diretamente proporcional com o aumento da renda dessa população. ${ }^{19}$

De acordo com o atual estudo, a proporção de idosos classificados com sobrepeso atingiu 41,78\%, enquanto 30,14\% da amostra apresentaram estado nutricional de obesidade, baseando-se nos parâmetros da WHO, indicando que a maior parte dos idosos da amostra (71,92\%) foi identificada com excesso de peso, semelhantemente ao estudo nacional da POF. ${ }^{7}$

Foi possível observar que há uma associação entre a presença de ansiedade e um maior IMC nessa amostra de idosos. Os indivíduos identificados com ansiedade apresentaram uma média maior 
de IMC que aqueles classificados como não ansiosos. Resultados semelhantes foram observados por outros autores.

Um estudo realizado em duas grandes pesquisas epidemiológicas, da população geral da Alemanha, cuja finalidade foi investigar transtornos mentais em pacientes com obesidade ( $\mathrm{n}=$ 910) em comparação com saudáveis $(n=495)$ e com sobrepeso $(n=1550)$, concluiu que há um aumento no risco de desenvolvimento de transtornos mentais em indivíduos obesos, não apenas para depressão, mas também para ansiedade e transtornos de humor..$^{20}$

Outro estudo realizado nos Estados Unidos com os resultados da Pesquisa Nacional Epidemiológica sobre o Álcool e condições relacionadas, ${ }^{21}$ avaliando uma amostra de 40.790 adultos com idade superior a 18 anos, apresentou o objetivo de examinar diferenças entre os gêneros na associação com IMC e transtornos afetivos. A prevalência de transtornos de ansiedade foi associada com maior IMC em ambos os sexos. Porém, mulheres tiveram taxas superiores às dos homens. ${ }^{21}$

Por outro lado, em um estudo com uma população representativa de adultos alemães, composta por 4.185 sujeitos entre 18 e 65 anos de idade, cujo objetivo foi examinar associações entre obesidade e determinantes sociodemográficos, problemas psicológicos e transtornos mentais, não houve diferenças para a maioria dos transtornos mentais entre indivíduos obesos e não obesos. ${ }^{22}$

Uma revisão sistemática da literatura com meta-análise, que utilizou os estudos citados acima, encontrou evidência moderada e associação positiva entre obesidade e ansiedade. Dos 16 estudos selecionados (2 longitudinais e 14 estudos transversais), mais da metade mostrou chances significativamente maiores da probabilidade de transtornos de ansiedade em obesos do que em indivíduos não obesos. ${ }^{23}$

A revisão trouxe a reflexão que o efeito causal da obesidade sobre o desenvolvimento de ansiedade não pode ser concluído com base em dados atuais. Na revisão também foi encontrada associação entre obesidade e ansiedade por gênero. Dos estudos que foram estratificados por sexo, mais da metade apresentou uma tendência positiva para mulheres. Em alguns estudos, houve uma tendência positiva para homens. ${ }^{23}$

Segundo Canetti et al., ${ }^{24}$ está amplamente aceito que o comportamento alimentar nos humanos altera-se de acordo com suas emoções, como ansiedade, raiva, alegria, depressão e tristeza. Emoções negativas têm sido estudadas e sugerem que elas podem influenciar o consumo de alimentos. ${ }^{24}$

Um estudo nacional realizado na Universidade de Brasília $(\mathrm{UnB})^{25}$ que avaliou as contribuições de aspectos psicológicos e nutricionais na mudança do comportamento alimentar, com uma amostra de 11 sujeitos, de ambos os sexos, na faixa etária entre 22 e 62 anos, identificou que fatores psicológicos interferem na prática de hábitos alimentares adequados. Sentimentos como tristeza, raiva, depressão, ansiedade, dificuldades no relacionamento familiar, baixa autoestima, 
avaliação negativa da imagem corporal, motivação e alegria, refletem na escolha alimentar. Foi constatado nessa amostra que depressão e ansiedade representaram as emoções mais frequentes. ${ }^{25}$

No entanto, a influência que as emoções podem causar no comportamento alimentar são mais fortes em pessoas obesas do que em indivíduos que não estão acima do peso. Isso tem sido explicado no estudo das teorias psicossomáticas da obesidade. ${ }^{24}$

De acordo com França et al., ${ }^{25}$ pessoas obesas, quando estavam ansiosas, comiam demais e isso reduzia a ansiedade. Essa relação poderia envolver efeitos diferenciados da proteína e carboidrato na síntese de neurotransmissores, como a serotonina. Indivíduos obesos também apresentam dificuldades de diferenciar a fome e a ansiedade, pois comem em resposta a ambas. ${ }^{25}$

De acordo com os estudos elencados, pôde-se observar que indivíduos obesos tinham maior propensão à ansiedade.

Alguns fatores podem ter influenciado nos achados da presente pesquisa, como o fato de a grande predominância (quase $90 \%$ dos idosos) pertencer ao sexo feminino. A mesma limitação ocorreu em outros estudos com diferentes abordagens e objetivos, como o realizado por Previato et al. ${ }^{26}$ em Outro Preto-MG, em que 71,4\% dos presentes eram mulheres idosas. ${ }^{26}$ Um outro trabalho realizado em Viçosa-MG apresentou elevada participação do sexo feminino, 84,4\%. ${ }^{27}$ Um terceiro, com predominância do sexo feminino, é o de Victor et al., ${ }^{28}$ feito em Fortaleza-CE, no qual 77,1\% dos participantes são mulheres idosas. ${ }^{28}$

Neste trabalho, entre os idosos classificados como ansiosos, 95\% são mulheres. Pode-se afirmar que a ansiedade foi mais presente no sexo feminino, uma vez que o estudo foi realizado em uma população específica, além da maior participação das mulheres na pesquisa. Outro ponto a ser destacado é o fato do NETI/UFSC possuir mais alunos do sexo feminino matriculados em relação ao sexo masculino. Dados do IBGE de 2003 indicaram que as mulheres são as que mais procuram atendimento médico, bem como referem maior prevalência de doenças crônicas no País. ${ }^{29}$

\section{Conclusão}

Este estudo evidenciou a associação entre a obesidade e a presença de ansiedade em uma população idosa de Florianópolis, SC. A maior parte desta população foi composta por mulheres, o que pode ter influenciado nos achados aqui descritos. Não obstante, ainda permanece obscuro o papel da obesidade na gênese dos transtornos afetivos, entre eles, a ansiedade.

A população mundial está em processo de envelhecimento. Dessa forma, é muito importante que sejam desenvolvidos mais estudos nesta área, para que dêem subsídios para a elaboração de políticas e programas de promoção e atenção à saúde mental do idoso, assim como programas e ações de promoção e prevenção do excesso de peso, que cada vez é mais frequente nesta população. 


\section{Agradecimentos}

Agradecemos à coordenadora do Núcleo de Estudos da Terceira Idade, Prof $\underline{a}$ Jordelina Schier, pela disponibilidade em auxiliar na execução desta pesquisa, e a toda a equipe do Núcleo de Estudos da Terceira Idade (NETI).

\section{Colaboradores}

De Matos PP e Ribeiro SV participaram da idealização do projeto de pesquisa, execução e coleta de dados, análise estatística e redação do manuscrito. Moreira JD, da idealização do projeto de pesquisa, análise estatística, redação do manuscrito e orientação e supervisão das acadêmicas.

Conflito de interesses: Os autores declaram não haver conflito de interesses.

\section{Referências}

1. Karg RS, Bose J, Batts KR, Forman-Hoffman VL, Liao D, Hirsch E, et al. Past year mental disorders among adults in the United States: results from the 2008-2012 Mental Health Surveillance Study. CBHSQ Data Review Oct. 2014. Disponível em: https://www.ncbi.nlm.nih.gov/books/NBK379142/ pdf/Bookshelf_NBK379142.pdf

2. Valiengo LC, Stella F, Forlenza OV. Mood disorders in the elderly: prevalence, functional impact, and management challenges. Neuropsychiatr Dis Treat. 2016; 12:2105-2114.

3. Brasil. Ministério da Saúde. Secretaria de Atenção à Saúde. Departamento de Atenção Básica. Envelhecimento e Saúde da Pessoa Idosa. Brasília: Ministério da Saúde; 2006. Cadernos de Atenção Básica $n^{\circ} 19$. Série A. Normas e manuais técnicos.

4. Suma C, Chaitanya R, Jothika M. Anxiety disorders in older adults. Geriatric Psychiatry 2014; 3:273-281.

5. Santa Catarina. Secretariade Estado da Saúde. Transtorno de Pânico - Protocolo Clínico. [acesso em: 07 mar. 2017. Disponível em: http://portalses.saude.sc.gov.br/index.php?option=com_conte nt\&view $=$ article\&id=5313:protocolos-da-rede-de-atencao-psicossocial\&catid=1019:protocolos-ediretrizes-terapeuticas

6. Ogden CL, Carrol MD, Kit BK, Flegal, KM. Prevalence of childhood and adult obesity in the united states. Jama 2014; 8(311):806-814.

7. Instituto Brasileiro de Geografia e Estatística. IBGE. Sinopse do censo demográfico [Internet] 2010. [acesso em: 10 nov. 2015]. Disponível em: http://www.censo2010.ibge.gov.br/sinopse/index. php?dados $=12 \& u f=00$

8. Associação Brasileira para o Estudo da Obesidade e da Síndrome Metabólica. Diretriz brasileira de obesidade, 2016. $4^{\text {a }}$ ed. São Paulo: ABESO; 2016. 
9. Simon GE, Von Korff M, Saunders K, Miglioretti DL, Crane PK, Van Belle G, et al. Association between obesity and psychiatric disorders in the US adult population. Arch Gen Psychiatry 2016; 63(7):824-830.

10. Torres SJ, Nowson CA. Relationship between stress, eating behavior, and obesity. Nutrition 2007; 23(11-12):887-894.

11. Pachana NA, Byrne GJ, Siddle H, Koloski N, Harley E, Arnold E. Development and validation of the Geriatric Anxiety Inventory. Int Psychogeriatr. 2007; 19(1):103-114.

12. Martiny C, Silva ACO, Nardi AE, Pachana NA. Tradução e adaptação transcultural da versão brasileira do Inventário de Ansiedade Geriátrica (GAI). Rev Psiq Clín. 2011; 38(1):08-12.

13. Versiani M. Princípios gerais básicos das escalas de avaliação. In: Centro de Pesquisa em Psicobiologia Clínica. editor. Escalas de avaliação para monitorização de tratamentos com psicofármacos. São Paulo: Associação Fundo de Incentivo à Psicofarmacologia; 1989.

14. Associação Psiquiátrica Americana. Manual diagnóstico e estatístico de transtornos mentais. DSMIV-TR. Porto Alegre: Artmed; 2003.

15. World Health Organization. The world health report: primary health care now more than ever. Geneva: WHO; 2008. 119 p.

16. Lipschitz DA. Screening for nutritional status in the elderly. Prim Care 1994; 21:55-67.

17. Brasil. Ministério da Saúde. Secretaria de Vigilância em Saúde. Secretaria de Atenção à Saúde. Política Nacional de Promoção da Saúde. 3. ed. Brasília: Ministério da Saúde; 2010.

18. Wolitzky-Taylor KB, Castriotta N, Lenze EJ, Stanley MA, Craske MG. Anxiety disorders in older adults: a comprehensive review. Depress Anxiety 2010; 27:190-211.

19. Pereira Ingrid Freitas da Silva, Spyrides Maria Helena Constantino, Andrade Lára de Melo Barbosa. Estado nutricional de idosos no Brasil: uma abordagem multinível. Cad Saúde Pública 2016; 32(5):e00178814.

20. Baumeister H, Harter M. Mental disorders in patients with obesity in comparison with healthy probands. Int J Obes. 2007; 31:1155-1164.

21. Barry D, Pietrzak RH, Petry NM. Gender differences in associations between body mass index and DSM-IV mood and anxiety disorders: results from the national epidemiologic survey on alcohol and related conditions. Ann Epidemiol. 2008; 18:458-466.

22. Hach I, Ruhl UE, Klose M, Klotsche J, Kirch W, Jacobi F. Obesity and the risk for mental disorders in a representative German adult sample. Eur J Public Health 2007; 17:297-305.

23. Gariepy G, Nikita D, Schimitz N. The association between obesity and anxiety disorders in the population: a systematic review and meta- analysis. Int J Obes. 2010; 34(3):407-419.

24. Canetti L, Bachar E, Berry EM. Food and emotion. Behav Processes 2012; 60:157-164.

25. França CL, Biaginni M, Mudesto APL, Alves ED. Contribuições da psicologia e da nutrição para a mudança do comportamento alimentar. Estud Psicol. 2012; 17(2):337-345. 
26. Previato H, Barros F, Mello J, Silva F, Nimer M. Perfil clínico-nutricional e consumo alimentar de idosos do programa terceira idade. DEMETRA: Alimentação, Nutrição \& Saúde. 2015; 10(2): 375-387.

27. Ferreira PM, Rosado GP. Perfil de usuários e percepção sobre a qualidade do atendimento nutricional em um programa de saúde para a terceira idade. Rev Bras Geriatr Gerontol. 2012; 15(2):243-254.

28. Victor JF, Ximenes LB, Almeida PC, Vasconcelos FF. Perfil sociodemográfico e clínico de idosos atendidos em Unidade Básica de Saúde da Família. Acta Paul Enferm. 2009; 22(1):49-54.

29. Instituto Brasileiro de Geografia e Estatística. Pesquisa Nacional por Amostra de Domicílios. Síntese de indicadores sociais [internet] 2003 [citado em: 23 jun. 2017]. Disponível em: http://biblioteca.ibge. gov.br/index.php/biblioteca-catalogo? view $=$ detalhes\&id $=21882$ 
